\title{
Actual curriculum development practices instrument: testing for factorial validity
}

\begin{abstract}
The Actual Curriculum Development Practices Instrument (ACDP-I) was developed and the factorial validity of the ACDP-I was tested $(n=107)$ using exploratory factor analysis procedures in the earlier work of [1]. Despite the ACDP-I appears to be content and construct valid instrument with very high internal reliability qualities for using in Malaysia, the accumulated evidences are still needed to provide a sound scientific basis for the proposed score interpretations. Therefore, the present study addresses this concern by utilising the confirmatory factor analysis to further confirm the theoretical structure of the variable Actual Curriculum Development Practices (ACDP) and enrich the psychometrical properties of ACDP-I. Results of this study have practical implication to both researchers and educators whose concerns focus on teachers' classroom practices and the instrument development and validation process.
\end{abstract}

Keyword: Instrument development; Confirmatory factor analysis; Construct validation; Actual curriculum 